\title{
Challenges and Controversies in Translational Stroke Research - an Introduction
}

\author{
Johannes Boltze ${ }^{1,2} \cdot$ Cenk Ayata ${ }^{3,4,5}$
}

Received: 2 August 2016 / Accepted: 4 August 2016/Published online: 31 August 2016

(C) Springer Science+Business Media New York 2016

Translational stroke research is at a turning point. Of countless efficacious interventions reported in animal models of stroke, few if any have proven unequivocally efficacious in clinical trials, and none has entered clinical practice since thrombolysis [1]. Despite the widened therapeutic windows for systemic and endovascular thrombolysis, more than $90 \%$ of acute stroke victims do not benefit from therapeutic options directly limiting the ischemic injury and improving the functional outcome [2]. Therefore, the translational roadblock is still standing [3]. As we explore the reasons for failure and consider novel strategies to enhance the predictability and clinical relevance of preclinical stroke research, we must be prepared to challenge the scientific methodology and even the most fundamental concepts in stroke pathophysiology. This special edition collects papers from renowned experts in the field on topics that are likely to shape the next generation of translational stroke research.

Cenk Ayata

cayata@mgh.harvard.edu

1 Fraunhofer Institute for Cell Therapy and Immunology, Leipzig, Germany

2 Fraunhofer Research Institution for Marine Biotechnology and Institute for Medical and Marine Biotechnology, University of Lübeck, Lübeck, Germany

3 Neurovascular Research Lab, Department of Radiology, Massachusetts General Hospital, Harvard Medical School, Charlestown, MA, USA

4 Stroke Service and Neuroscience Intensive Care Unit, Department of Neurology, Massachusetts General Hospital, Harvard Medical School, Boston, MA, USA

5 Neuroscience Center, Neurovascular Research Laboratory, 149 13th Street, Charlestown, MA 02129, USA
Over the recent years, stroke research has seen fundamental methodological improvements [4]. The concept of ultrastandardized stroke modeling is challenged by the inherent variability of stroke in patients, increasingly considered an essential element of human condition. Kent and Mandava suggest an entirely new approach to stroke modeling by considering stroke severity and other baseline factors on outcome, and embrace variability. They utilize statistical methods developed for clinical investigations to strengthen the translational power of their approach [5]. Ergul et al. emphasize the importance of modeling prevalent stroke comorbidities, hypertension and diabetes, when considering stroke complexity [6] while Ahnstedt and co-workers provide a strong rationale for investigating sex differences in translational stroke research [7, 8]. Hoffmann et al. describe confounding effects of anesthesia in general, and the specific impact of different anesthetics on stroke outcome [9]. Yet, implementing all relevant factors can hardly be achieved by a single center and will result in inter-study heterogeneities most likely prohibiting clear statistical results with common sample sizes. The research community is examining and debating concepts derived from clinical research such as the new preclinical "phase 3 " trial platforms to meet these challenges [10, 11]. Boltze et al. summarize the community opinion on multicenter ("phase 3") preclinical trials, a new concept for confirmative stroke research [12]. Last but not the least, we need to redefine the academic-industry collaborations in translational stroke research, which are sometimes impeded by simplistic research strategies or by focusing on improper readout parameters [13]. Those may initially limit study costs, but can lead to falsenegative results.

Intelligent endpoint selection is another key element in planning experimental as well as clinical studies. Functional endpoints are considered indispensible in preclinical stroke research, but do not necessarily predict clinical outcomes [14]. The issue is even more pressing in stroke recovery 
research where tissue endpoints are scarce. The correspondence between clinical and preclinical functional outcomes must hence be examined and enhanced. New concepts on how recovery should be measured preclinically (e.g., by a tailored latent variable approach) are suggested by Hommel et al. [15] while Jolkkonen and Kwakkel suggest to tackle confounders and sources of study result variability [16]. Muir and Macrae show that multimodal neuroimaging as a clinically relevant outcome surrogate can improve accuracy and statistical power in smaller samples and is a meaningful preclinical trial recruitment tool [17]. It further enables repeated longitudinal assessments of brain structure, perfusion, and metabolism, representing best-correlating endpoints to neurological function.

The relevance of the central nervous and the immune "super" systems' interplay in stroke pathophysiology and outcome is increasingly recognized [18]. It provides novel targets for therapeutic interventions. For instance, the interleukin-1 receptor antagonist (IL-1RA), already applied in clinical rheumatology, is of direct relevance for research programs targeting human stroke by means of immunomodulation. McCann and colleagues assessed preclinical study quality and reported outcomes. Their analyses clearly support the IL-1RA treatment concept for stroke. They note that the quality of preclinical experiments has significantly increased as compared to previous research programs [19]. Dr. Becker describes inter-strain and inter-species differences in immune responses to stroke among frequently used animal models [20]. Liesz and Kleinschnitz review the role of regulatory $\mathrm{T}$ cells $\left(\mathrm{T}_{\text {regs }}\right)$ on the secondary inflammatory response after stroke, as well as different $T_{\text {reg }}$ sites of action such as the neurovascular unit and the surrounding inflammatory milieu [21]. Closely related, new and better experimental models of vascular cognitive impairment are needed as pointed out by Yang et al. [22]. Immune responses may play a substantial role [23].

Cell-based therapies are at the cutting edge of experimental stroke treatment, currently being translated in early stage clinical studies [24]. Autologous transplantation of adult cell populations such as bone marrow mononuclear cells (BM MNC) is most frequently applied. The group of Dr. Savitz investigated the therapeutic impact of BM MNC subfractions after stroke, bringing clarity into the therapeutic mode of action [25]. Rodríguez-Frutos and colleagues point out that successful clinical implementation of cell therapies hinges on several factors, including safe and efficacious routes for cell administration [26].

Although advances in mechanical recanalization have recently ended two decades of drought in stroke therapy development [27], still only a small fraction of stroke victims benefit from it. The next natural step is to develop strategies that widen the therapeutic window for recanalization therapies as shown by Henninger and Fisher [2]. Linfante and Cipolla suggest combination therapies taking advantage of endovas cular thrombolysis [28] in order to include more patients. At the same time, new concepts overcoming existing contraindications must be developed.

While all topics discussed in this special issue are specifically relevant for stroke, many are also of general translational importance for the entire field of experimental neuroscience. By providing new ideas on current challenges and controversies, the contributions, concepts, and considerations herein ultimately aim to help overcome the translational roadblock.

\section{Compliance with Ethical Standards}

Conflict of Interest The authors declare that they have no competing interests.

Funding Only institutional funds were used for this project.

\section{References}

1. O'Collins VE, Macleod MR, Donnan GA, Horky LL, van der Worp BH, Howells DW. 1,026 experimental treatments in acute stroke. Ann Neurol. 2006;59(3):467-77.

2. Henninger N, Fisher M. Extending the time window for endovascular and pharmacological reperfusion. Transl Stroke Res. 2016;7(4):284-93.

3. Endres M, Engelhardt B, Koistinaho J, Lindvall O, Meairs S, Mohr JP, et al. Improving outcome after stroke: overcoming the translational roadblock. Cerebrovasc Dis. 2008;25(3):268-78.

4. Fisher M, Feuerstein G, Howells DW, Hurn PD, Kent TA, Savitz SI, et al. Update of the stroke therapy academic industry roundtable preclinical recommendations. Stroke. 2009;40(6):2244-50.

5. Kent TA, Mandava P. Embracing biological and methodological variance in a new approach to pre-clinical stroke testing. Transl Stroke Res. 2016;7(4):274-83.

6. Ergul A, Hafez S, Fouda A, Fagan SC. Impact of comorbidities on acute injury and recovery in preclinical stroke research: focus on hypertension and diabetes. Transl Stroke Res. 2016;7(4):248-60.

7. Ahnstedt H, McCullough LD, Cipolla MJ. The importance of considering sex differences in translational stroke research. Transl Stroke Res. 2016;7(4):261-73.

8. Ahnstedt H, Sweet J, Cruden P, Bishop N, Cipolla MJ. Effects of early post-ischemic reperfusion and tPA on cerebrovascular function and nitrosative stress in female rats. Transl Stroke Res. 2016;7(3):228-38.

9. Dirnagl U, Hakim A, Macleod M, Fisher M, Howells D, Alan SM, et al. A concerted appeal for international cooperation in preclinical stroke research. Stroke. 2013;44(6):1754-60.

10. Hoffmann U, Sheng H, Ayata C, Warner DS. Anesthesia in experimental stroke research. Transl Stroke Res. 2016. doi:10.1007 /s12975-016-0491-5.

11. Boltze J, Ayata C, Wagner DC, Plesnila N. Preclinical phase III trials in translational stroke research: call for collective design of framework and guidelines. Stroke. 2014;45(2):357. 
12. Boltze J, Wagner DC, Henninger N, Plesnila N, Ayata C. Phase III preclinical trials in translational stroke research: community response on framework and guidelines. Transl Stroke Res. 2016;7(4):241-7.

13. Boltze J, Wagner DC, Barthel H, Gounis MJ. Academic-industry collaborations in translational stroke research. Transl Stroke Res. 2016;7(4): 343-53.

14. Macleod MR, van der Worp HB, Sena ES, Howells DW, Dirnagl U, Donnan GA. Evidence for the efficacy of NXY-059 in experimental focal cerebral ischaemia is confounded by study quality. Stroke. 2008;39(10):2824-9.

15. Hommel M, Detante O, Favre I, Touzé E, Jaillard A. How to measure recovery? Revisiting concepts and methods for stroke studies. Transl Stroke Res. 2016. doi:10.1007/s12975-016-0488-0.

16. Jolkkonen J, Kwakkel G. Translational hurdles in stroke recovery studies. Transl Stroke Res. 2016;7(4):331-42.

17. Muir KW, Macrae IM. Neuroimaging as a selection tool and endpoint in clinical and pre-clinical trials. Transl Stroke Res. 2016. doi:10.1007/s12975-016-0487-1.

18. Benakis C, Brea D, Caballero S, Faraco G, Moore J, Murphy M, et al. Commensal microbiota affects ischemic stroke outcome by regulating intestinal $\gamma \delta$ T cells. Nat Med. 2016;22(5):516-23.

19. McCann SK, Cramond F, Macleod MR, Sena ES. Systematic review and meta-analysis of the efficacy of interleukin-1 receptor antagonist in animal models of stroke: an update. Transl Stroke Res. 2016. doi:10.1007/s12975-016-0489-z.

20. Becker KJ. Strain-related differences in the immune response: relevance to human stroke. Transl Stroke Res. 2016;7(4):303-12.
21. Liesz A, Kleinschnitz C. Regulatory T cells in post-stroke immune homeostasis. Transl Stroke Res. 2016;7(4):313-21.

22. Yang Y, Kimura-Ohba S, Thompson J, Rosenberg GA. Rodent models of vascular cognitive impairment. Transl Stroke Res. 2016. doi:10.1007/s12975-016-0486-2.

23. Kaiser D, Weise G, Möller K, Scheibe J, Pösel C, Baasch S, et al. Spontaneous white matter damage, cognitive decline and neuroinflammation in middle-aged hypertensive rats: an animal model of early-stage cerebral small vessel disease. Acta Neuropathol Commun. 2014;2:169.

24. Janowski M, Wagner DC, Boltze J. Stem cell-based tissue replacement after stroke: factual necessity or notorious fiction? Stroke. 2015;46(8):2354-63.

25. Yang B, Parsha K, Schaar K, Xi X, Aronowski J, Savitz SI. Various cell populations within the mononuclear fraction of bone marrow contribute to the beneficial effects of autologous bone marrow cell therapy in a rodent stroke model. Transl Stroke Res. 2016;7(4): 322-30.

26. Rodríguez-Frutos B, Otero-Ortega L, Gutiérrez-Fernández M, Fuentes B, Ramos-Cejudo J, Díez-Tejedor E. Stem cell therapy and administration routes after stroke. Transl Stroke Res. 2016. doi:10.1007/s12975-016-0482-6.

27. Khandelwal P, Yavagal DR, Sacco RL. Acute ischemic stroke intervention. J Am Coll Cardiol. 2016;67(22):2631-44.

28. Linfante I, Cipolla MJ. Improving reperfusion therapies in the era of mechanical thrombectomy. Trans1 Stroke Res. 2016;7(4):294-302. 